\title{
Can portal hypertension and hepatic decompensation be predicted?
}

\author{
Kosuke Kaji ${ }^{1}$ Hitoshi Yoshiji ${ }^{1}$
}

Received: 29 December 2019/ Accepted: 9 January 2020/Published online: 21 January 2020

(C) Japanese Society of Gastroenterology 2020

Keywords Liver cirrhosis · Hepatic decompensation .

Portal hypertension

$\begin{array}{ll}\text { Abbreviations } \\ \text { CSPH } & \text { Clinically significant portal hypertension } \\ \text { VWF-Ag } & \text { Von Willebrand factor antigen } \\ \text { VITRO } & \text { The ratio of VWF-Ag to platelets } \\ \text { HVPG } & \text { Hepatic venous pressure gradient } \\ \text { TE } & \text { Transient elastography } \\ \text { MELD } & \text { Model for end-stage liver disease } \\ \text { SVR } & \text { Sustained virologic response } \\ \text { HCV } & \text { Hepatitis C }\end{array}$

Liver cirrhosis is histologically characterized by diffuse nodular regeneration, collapsed liver structures, and prominent distortion of the hepatic vascular system [1]. The alterations of the hepatic architecture coincide with increased portal vascular resistance, evoking portal hypertension. Clinically significant portal hypertension (CSPH) is a key manifestation of cirrhosis progression to decompensation, because it is pathologically associated with essential cirrhotic complications, such as bleeding gastroesophageal varices, pancytopenia with hypersplenism, hepatic encephalopathy, massive ascites, and hepatorenal syndrome. These complications substantially impair the prognosis and survival rate of cirrhotic patients;

Kosuke Kaji

kajik@naramed-u.ac.jp

1 Department of Gastroenterology, Nara Medical University, Kashihara, Nara 634-8521, Japan hence, the severity of CSPH is an indispensable factor when attempting to predict progression to a decompensated state and mortality among cirrhotic patients [1].

Present global guidelines recommend measurement of the hepatic venous pressure gradient (HVPG) as a diagnostic indicator of CSPH [2]. However, HVPG is measured by moderately invasive interventional radiology. Thus far, transient elastography (TE) may enable the identification of patients at risk for CSPH and decompensation. The Baveno VI consensus conference has recommended that patients with a TE $<20 \mathrm{kPa}$ and a platelet count $>150 \times 10^{9} / \mathrm{L}$ can be excluded from high-risk gastroesophageal varices with high significant negative predictive value [2]. TE is a non-invasive procedure but not universally available, hence there is a requirement for novel and more conveniently measured surrogate markers that reflect the severity of CSPH.

In the issue of the "Journal of Gastroenterology", Schwarzer et al. reported their analysis of the validity of VITRO, i.e., the ratio of von Willebrand factor antigen (VWF-Ag) to platelets, as a prognostic marker of hepatic decompensation and mortality in patients with compensated cirrhosis [3]. They demonstrated that cirrhotic patients with a VITRO score [plasma levels of VWF-Ag $(\%) /$ platelet count $\left.\left(10^{9} / \mathrm{L}\right)\right] \geq 2.5$ at baseline showed a three-times higher incidence of progression to a decompensated state at the 45-month follow-up [3]. Moreover, this issue revealed that the patients with a VITRO score $\geq 2.5$ were likely to show a lower transplant-free survival rate, implying a higher risk of liver-related death [3]. Several clinical studies also have reported the potential of inflammatory and/or fibrotic component-related markers to predict CSPH in cirrhotic patients. Buck et al. suggested that the inflammatory biomarkers such as IL-1 $\beta$, IL-1R $\alpha$, Fas-R, VCAM-1, TNF $\beta$, and HSP70 showed significant 
correlation with HVPG measurement in a compensated cirrhosis cohort [4]. Wang et al. investigated the predictive performances of seven fibrosis indexes, the aspartate aminotransferase (AST)-to-alanine aminotransferase (ALT) ratio (AAR), AST-to-platelet ratio index (APRI), fibrosis index (FI), FIB-4, Forns index, King's score and the Lok index, and they concluded that the combined King's score with the Lok index might be useful for an initial screening of cirrhotic patients with higher risk of CSPH [5]. Moreover, Sandahl et al. demonstrated that the combination of the macrophage-specific activation marker sCD163 with ELF fibrosis score consisting of hyaluronic acid, tissue inhibitor of metalloproteinase-1, and procollagen-III aminopeptide accurately predicted CSPH in patients with cirrhosis [6]. These evidences are supported by pathological concept that the increased intrahepatic perfusion resistance in portal hypertension is attributed to both an inflammatory and a fibrotic component in liver cirrhosis.

Furthermore, recent clinical studies have demonstrated that anti-hepatitis C (HCV) therapy-based sustained virologic response (SVR) is relevant to amelioration of portal hypertension [7, 8]. Meanwhile, changes in HVPG appear to be more heterogeneous in the patients with severe fibrosis, and Puigvehí et al. claimed that HCV-related cirrhotic patients could develop high-risk gastroesophageal varices after SVR [9]. These findings indicated the importance of continued monitoring of portal hypertension after HCV eradiation, especially for the patients with HCVrelated cirrhosis. VITRO scores were significantly decreased after SVR relative to baseline in a subgroup of patients with HCV-related cirrhosis [2]. In this respect, using VITRO scores might improve the distinction of patients with negligible risk from those with indispensable risk for decompensation after SVR, and further investigation is required to evaluate the changes in above CSPHpredictive biomarkers besides VITRO scores by HCV eradiation in cirrhotic patients.

Schwarzer et al. cogently argued for the use of the VITRO score as a novel surrogate marker to assess the risk of decompensation and mortality in conjunction with CSPH in patients with compensated cirrhosis [2]. Given this aspiration, several limitations of their clinical study are alluded to by the authors. First, the study was retrospectively designed and the majority of patients had HCV-related cirrhosis. Second, the correlation between the relative change of VITRO score and HVPG levels following HCV eradication was incompletely assessed because of the small sample size that was examined. Third, VWF-Ag levels are influenced by gut-derived endotoxin, which is often modified by systemic status and therapeutic interventions in cirrhotic patients [10]. These issues should be addressed in the near future to accomplish the full validation of the VITRO score as a predictor of the risk of decompensation and mortality in cirrhotic patients.

\section{Compliance with ethical standards}

Conflict of interest The authors declare that they have no conflict of interest.

\section{References}

1. Tsochatzis EA, Bosch J, Burroughs AK. Liver cirrhosis. Lancet. 2014;383:1749-61.

2. de Franchis R, Faculty BVI. Expanding consensus in portal hypertension: Report of the Baveno VI Consensus Workshop: Stratifying risk and individualizing care for portal hypertension. J Hepatol. 2015;63:743-52.

3. Schwarzer R, Reiberger T, Mandorfer M, et al. The von Willebrand Factor antigen to platelet ratio (VITRO) score predicts hepatic decompensation and mortality in cirrhosis. J Gastroenterol. 2019;5:5.

4. Buck M, Garcia-Tsao G, Groszmann RJ, et al. Novel inflammatory biomarkers of portal pressure in compensated cirrhosis patients. Hepatology. 2014;59:1052-9.

5. Wang L, Feng Y, Ma X, et al. Diagnostic efficacy of noninvasive liver fibrosis indexes in predicting portal hypertension in patients with cirrhosis. PLoS One. 2017;12:e0182969.

6. Sandahl TD, McGrail R, Møller HJ, et al. The macrophage activation marker sCD163 combined with markers of the enhanced liver fibrosis (ELF) score predicts clinically significant portal hypertension in patients with cirrhosis. Aliment Pharmacol Ther. 2016;43:1222-311.

7. Mandorfer M, Kozbial K, Schwabl P, et al. Sustained virologic response to interferon-free therapies ameliorates $\mathrm{HCV}$-induced portal hypertension. J Hepatol. 2016;65:692-9.

8. Lens S, Alvarado-Tapias E, Mariño Z, et al. Effects of all-oral anti-viral therapy on HVPG and systemic hemodynamics in patients with hepatitis C virus-associated cirrhosis. Gastroenterology. 2017;153:1273-83.

9. Puigvehí M, Londoño MC, Torras X, et al. Impact of sustained virological response with DAAs on gastroesophageal varices and Baveno criteria in HCV-cirrhotic patients. J Gastroenterol. 2019;5:5.

10. Carnevale R, Raparelli V, Nocella C, et al. Gut-derived endotoxin stimulates factor VIII secretion from endothelial cells. Implications for hypercoagulability in cirrhosis. $\mathbf{J}$ Hepatol. 2017;67:950-6.

Publisher's Note Springer Nature remains neutral with regard to jurisdictional claims in published maps and institutional affiliations. 